\title{
Las estrategias de comprensión textual y su eficacia en el aula
}

\author{
Adriana Castro-Pedraza* \\ Nidia Jazmín Páez-Castiblanco ${ }^{*}$
}

Estudiante de la Maestría en Lingüística, Universidad Pedagógica y Tecnológica de Colombia (UpTC). Profesora de lengua castellana y tutora del programa "Todos a Aprender", Ministerio de Educación Nacional.

Correo electrónico:

adrianacp0307@gmail.com

** Estudiante de la Maestría en Lingüística, Universidad Pedagógica y Tecnológica de Colombia (UPTC). Profesora ocasional "Seminarios de Investigación", UPTC. Correo electrónico: nidiapeti@hotmail.com

Recibido: 20 de mayo del 2015 Aprobado: 4 de agosto del 2015

Cómo citar este artículo: Castro-Pedraza, Adriana y Nidia Jazmín Páez-Castiblanco. "Las estrategias de comprensión textual y su eficacia en el aula". Rastros Rostros 17.31 (2015): 79-87. Impreso. doi: http://dx.doi. org/10.16925/ra.v17i31.1269

\section{Resumen}

Introducción: este artículo presenta los resultados de la investigación "El mundo de la lectura: estrategias para la comprensión”, adscrita a la línea Pedagogía del Lenguaje de la Maestría en Lingüística de la Universidad Pedagógica y Tecnológica de Colombia (UPTC), cuyo tema gira alrededor de las dificultades en comprensión lectora de los estudiantes de $4 .^{\circ}$ y $5 .^{\circ}$ de primaria, así como algunas alternativas de mejoramiento. Se desarrolló en la Institución Educativa Juan José Neira, en Machetá, Cundinamarca. Metodología: este estudio utilizó la metodología de investigación-acción-participación, y se llevó a cabo en tres fases: diagnóstico, diseño e implementación de la propuesta, y evaluación, cada una de las cuales contó con la participación de los docentes. Resultados: se diseñó una propuesta de intervención pedagógica orientada al mejoramiento de la comprensión de lectura de los estudiantes, mediante la construcción de unidades didácticas. Conclusiones: se vio un índice de mejoramiento considerable en las estrategias de comprensión empleadas por los estudiantes, lo cual fue posible establecer por medio de una prueba de comprensión aplicada durante la evaluación. Asimismo, se resalta el trabajo colaborativo de los docentes y la construcción de una guía que presenta estrategias de tipo general, las cuales se pueden aplicar en el aula frente a la comprensión de diferentes tipos de textos.

Palabras clave: comprensión textual, estrategias de comprensión, tipología textual, unidad didáctica. 


\title{
Text comprehension strategies and their effectiveness in the classroom
}

\begin{abstract}
Introduction: this article presents the results of a research project entitled "El mundo de la lectura: estrategias para la comprensión" [The world of reading: comprehension strategies] attached to the Language Pedagogy line of the Master's in Linguistics at the Universidad Pedagógica y Tecnológica de Colombia (UPTC), whose topic revolves around difficulties in reading comprehension of fourth and fifth graders and some improvement alternatives. It was conducted at the Institución Educativa Juan José Neira in Machetá, Cundinamarca, Colombia. Methodology: this study used the methodology of Participatory Action Research and was conducted in three phases: 1) diagnosis, 2) design, and 3) implementation of the proposal and assessment, each with the participation of teachers. Results: an educational intervention proposal aimed at improving reading comprehension of students by creating teaching units was designed. Conclusions: a significant improvement rate was evident in comprehension strategies used by the students, which could be established through a comprehension test applied during assessment. Moreover, the collaborative work of teachers and the construction of a guide presenting general strategies that can be applied in the classroom for understanding different types of texts are highlighted.
\end{abstract}

Keywords: text comprehension, comprehension strategies, textual typology, teaching unit.

\section{As estratégias de compreensão textual e sua eficácia}

\section{na sala de aulas}

\section{Resumo}

Introdução: este artigo apresenta os resultados a investigação titulada "O mundo da leitura: estratégias para a compreensão", adscrita à linha Pedagogia da Linguagem do Mestrado em Linguística da Universidade Pedagógica e Tecnológica da Colômbia (UPTC), cujo tema está focado em torno das dificuldades em compreensão leitora dos estudantes de $4^{\circ}$ e $5^{\circ}$ do ensino primário e de algumas alternativas de melhoramento; foi desenvolvida na Instituição Educativa Juan José Neira, em Machetá, Cundinamarca, na Colômbia. Metodologia: este estudo utilizou a metodologia de Investigação - Ação - Participação e foi executado em três fases: 1) diagnóstico, 2) desenho e 3) implementação da proposta e avaliação, cada uma delas com a participação dos docentes. Resultados: foi criada uma proposta de intervenção pedagógica voltada ao melhoramento da compreensão de leitura dos estudantes, através da construção de unidades didáticas. Conclusões: evidenciou-se um índice de melhoramento notável nas estratégias de compreensão empregadas pelos estudantes, o que foi estabelecido através de um teste de compreensão aplicada durante a avaliação. Igualmente, destaca-se o trabalho colaborativo dos docentes e a construção de um guia que apresenta estratégias de tipo geral que podem ser aplicados na sala de aula perante a compreensão de diferentes tipos de textos.

Palavras-chave: compreensão textual, estratégias de compreensão, tipologia textual, unidade didática. 


\section{Introducción}

Uno de los grandes retos de la escuela es que los niños aprendan a leer correctamente. Maestros y padres de familia esperan que el estudiante, al terminar el nivel de básica primaria, alcance un nivel adecuado en la habilidad de lectura. Por otra parte, podría pensarse que toda persona escolarizada sabe leer. Sin embargo, es un hecho que esta pretensión no siempre se logra, ocasiona dificultades en el proceso de aprendizaje y repercute de forma negativa sobre la vida académica y social de los niños.

La concepción que el maestro tiene sobre lo que significa leer es un factor importante a la hora de ejercer la labor pedagógica, ya que se corre el riesgo de centrar la atención en la decodificación o la lectura fluida, lo cual es importante pero no suficiente, a fin de que el estudiante adquiera las habilidades necesarias que le permitan interpretar y asignar significado a los diversos textos a los que se enfrenta. De acuerdo con Solé, "leer es un proceso mediante el cual se comprende el lenguaje escrito, en esta comprensión interviene tanto el texto, su forma y contenido, como el lector, sus expectativas y conocimientos previos" (18). Este concepto de lectura revela la importancia del lector como sujeto activo e implica un acompañamiento pedagógico, de manera que esto fortalezca estrategias propias de cada individuo en relación con el tipo de texto que lee.

La lectura es un medio para alcanzar aprendizajes, es una herramienta que facilita la adquisición de conocimiento y, por lo tanto, compromete al docente en la búsqueda de acciones que le permitan al niño el desarrollo de estrategias de comprensión frente a los textos que lo rodean. Es así como se origina un proceso de investigación cuyo objetivo es diseñar una propuesta de intervención pedagógica, orientada al mejoramiento de la comprensión de lectura de los estudiantes mediante la construcción de unidades didácticas, la cual tiene en cuenta la experiencia y los conocimientos de los docentes de primaria de las sedes rurales y urbanas de una institución educativa oficial del departamento de Cundinamarca.

La estrategia de planeación se organizó alrededor de unidades didácticas integradoras, en las que se vincularon diferentes tipos de texto y áreas de conocimiento, al considerar que "la enseñanza de la lectura no es cuestión de un curso o un profesor, sino que es una cuestión de escuela, de proyecto curricular y de todas las materias en las que interviene" (Solé 14).
De esta manera, se promueve el trabajo colaborativo entre docentes, así como se resalta la transversalidad de la lectura no solo en la escuela, sino también en las actividades de la vida cotidiana.

Durante el trabajo de planeación y ejecución de las unidades didácticas se observó la respuesta de los estudiantes, la reflexión y acompañamiento del docente, la pertinencia de los textos utilizados y la eficacia de las acciones propuestas, con el fin de analizar algunos de los factores que determinan el éxito o fracaso de la intervención pedagógica.

Como producto del proceso investigativo se construyó una guía de orientación para el maestro, la cual pretende establecer estrategias generales de apoyo en el aula frente a las dificultades en comprensión textual que suelen presentarse en los últimos grados del nivel de básica primaria.

\section{La comprensión textual y sus implicaciones pedagógicas}

La lectura se consideró por mucho tiempo como la habilidad para descifrar un código escrito. Se podría decir que un buen lector era aquel que utilizaba la entonación adecuada y la pronunciación correcta al leer un texto, razón por la cual en la labor del docente se dio gran importancia a la decodificación de las palabras y su recitado en voz alta, de modo que hacia allí se dirigía los objetivos de enseñanza. Sin embargo, un estudiante puede leer muy bien en voz alta y no comprender el significado de un texto, lo cual indica que la mera decodificación no es suficiente para establecer si se sabe o no leer; es necesario tener en cuenta la comprensión textual.

De acuerdo con Mendoza:

La comprensión lectora ya no puede, pues, ser entendida solo como una labor de descodificación, traducción o reconocimiento de palabras y frases, como si lo único importante fuera el texto. Se trata más bien de un proceso interactivo entre la acción del sujeto y el contenido del texto. (229)

Considerar la lectura desde este punto de vista tiene implicaciones a nivel pedagógico, pues si bien una decodificación adecuada es un primer paso, el papel del maestro será movilizar los procesos cognitivos del estudiante y todos los conocimientos que posee, de manera que pueda asignarle significado no solo a un texto escrito, sino a la multiplicidad de textos que lo rodean. 
La activación de los conocimientos previos le permite al estudiante despertar su interés y encontrar razones para la lectura de un texto, más allá de sus obligaciones académicas. Lo anterior considerando que "leer es un proceso de interacción entre el lector y el texto, proceso mediante el cual el primero intenta satisfacer los objetivos que guían su lectura" (Solé 17). De allí la importancia tanto del texto como del lector, ya que se considera este último un agente activo que aporta en la construcción de sentido, al generar predicciones e inferencias que rechaza o comprueba de acuerdo con los propósitos que tiene al leer.

Esta interpretación es coherente con el modelo interactivo de la comprensión lectora, el cual identifica el equilibrio que debe haber entre lo que dice el texto y la interpretación que el lector hace de él, de manera que reconoce los aportes del lector en el proceso de interpretación a partir de los conocimientos que posee, integrándolos con la nueva información que suministra el texto. Según Mendoza, "el modelo interactivo ve la lectura como una actividad cognitiva compleja y al lector como un procesador activo de la información que contiene el texto" (234). Es decir, el lector es un sujeto activo del proceso, de acuerdo con sus características particulares y el contexto cognitivo en el que se da la lectura, pero no se aparta del contenido del texto, buscando comprender lo que quiso decir el autor a través de él.

La comprensión textual no se desarrolla de la misma forma en todos los individuos, en razón a las características particulares de cada uno. Es por eso que los maestros deben reconocer a qué nivel llegan sus estudiantes mediante diferentes estrategias de evaluación. Pérez señala que se pueden identificar tres niveles de comprensión lectora: literal, inferencial y crítico intertextual. El nivel literal se alcanza cuando se logra dar cuenta de los sujetos, objetos y eventos, así como del significado de frases y palabras, ya sea por literalidad transcriptiva o bien mediante la paráfrasis. El nivel inferencial se caracteriza por la capacidad de extraer información que no se encuentra explícita en el texto, lo cual requiere establecer relaciones de diverso tipo y supone una comprensión global. En el nivel crítico intertextual, el lector es capaz de tomar posición frente a lo que dice el texto, a fin de identificar su intencionalidad y reconocer su relación con el contexto y con otros textos.

Estos niveles de comprensión se pueden identificar en cada grado de escolaridad a través de actividades que involucren una variada tipología textual. Esto en la medida en que el uso de diferentes clases de textos le permite al estudiante generar estrategias de lectura apropiadas a lo que lee. De acuerdo con Solé, el hecho de trabajar distintos tipos de textos "nos hace estar alerta, nos hace esperar unos contenidos y no otros, nos permite actualizar ciertas estrategias y nos prepara para una lectura más ágil y productiva y para una mejor comprensión" (72). Es por esta razón que se propone que en las aulas se trabajen textos de tipo informativo, narrativo, explicativo y argumentativo, solo por mencionar la tipología textual propuesta por el Ministerio de Educación Nacional (1998), sin desconocer que existen otras posibilidades de clasificación.

$\mathrm{Al}$ ser la lectura un proceso cognitivo complejo, es previsible que surjan dificultades de diferente tipo, las cuales impiden al estudiante alcanzar los niveles de comprensión esperados. De acuerdo con el modelo interactivo, Arias sostiene cómo los factores que afectan el proceso lector pueden estar relacionados con alguno de los dos componentes: con el lector o con el material que se lee.

En relación con el lector - sujeto activo del proceso de lectura-, se presentan dificultades en relación con la activación de su conocimiento previo, el interés que despierta el texto, los propósitos que guían su lectura, su habilidad de decodificación y el uso de estrategias metacognitivas. Estos factores se deben identificar con el fin de establecer cuáles son las estrategias más eficientes para reducir las dificultades que tiene el lector.

En cuanto al material que se lee, se presentan interferencias relacionas con la cantidad de vocabulario desconocido que presenta, la longitud y complejidad de las frases, el nivel sintáctico que desarrolla y la forma como integra la información sobre un evento, objeto o personaje. De allí la importancia de que la persona encargada de orientar el proceso de comprensión lectora utilice textos apropiados para la edad y el nivel del niño, procurando variedad en la tipología textual en un nivel progresivamente más complejo.

Frente a las anteriores consideraciones, el rol del docente cobra un papel muy importante, pues su responsabilidad es promover estrategias de comprensión, las cuales le brinden al estudiante herramientas que le permitan un acercamiento inteligente a la forma más adecuada de realizar una tarea. Solé afirma que la enseñanza de estrategias de lectura en el aula permite "hacer lectores autónomos, capaces de enfrentarse de manera inteligente a textos de muy distinta índole, la mayoría de las veces distintos de 
los que se usan cuando se instruye" (61). Esto le permite al estudiante seleccionar o rechazar determinadas acciones a fin de facilitar la comprensión de un texto en particular, de modo que no se pondrán en juego las mismas estrategias frente a la lectura de una narración, que frente a la lectura de un afiche.

De forma general, las estrategias cognitivas que se pueden trabajar en las aulas de clase se refieren a tener claridad sobre los objetivos o propósitos de la lectura, la activación de conocimientos previos, identificar la información fundamental y descartar la menos relevante, comparar las expectativas con el contenido del texto, hacer inferencias de todo tipo y autorregular el proceso de comprensión. Sin embargo, para que estas estrategias sean eficientes, se deben emplear antes, durante y después de realizar la lectura. Antes de leer, permiten crear un contexto cognitivo apropiado para recibir la información del texto; durante la lectura tienen como finalidad promover la autorregulación de la comprensión; y, después de leer, contribuyen al desarrollo del proceso lector y no solo para verificar el nivel de comprensión que se alcanzó.

En síntesis, la comprensión lectora es un proceso de interacción entre texto y lector, con las dificultades e interferencias que cada uno representa. En la etapa escolar este proceso es trascendental pues marca el éxito o fracaso del aprendizaje de los niños, no solo en el contexto académico sino fuera de él, por lo cual se deben promover en el aula estrategias de comprensión eficientes frente a las dificultades que se pueden presentar.

\section{Metodología}

Esta investigación se llevó a cabo en el ámbito educativo con las características propias del enfoque cualitativo, mediante el uso de la metodología de la investigación acción participativa, cuya importancia "radica en que la misma comunidad, a través del descubrimiento de un problema con sus causas y consecuencias, comienza un proceso de concientización que no siempre estaba presente en la población afectada" (Bautista 97). Tal es el caso de la institución educativa Juan José Neira, en la cual los docentes identifican dificultades en la comprensión textual de los estudiantes de cuarto y quinto de la sede urbana y las diez sedes rurales, y deciden participar en la búsqueda de soluciones.

El proceso investigativo se desarrolló en tres fases: diagnóstico, diseño e implementación de la propuesta, y evaluación de los resultados. Durante la fase de diagnóstico, se aplicó una entrevista a los docentes, una encuesta a los estudiantes y una prueba de comprensión textual. Esto con el fin de identificar las dificultades específicas de los niños y determinar posibles causas. En la segunda fase, se trabajó con los docentes sobre las alternativas de mejoramiento frente a los resultados que se obtuvieron en la primera fase, estrategias estas que se organizaron en unidades didácticas alrededor de las diferentes tipologías textuales. A medida que se construyeron estas unidades, los maestros de cuarto y quinto las aplicaron con sus estudiantes, con el acompañamiento de las investigadoras. En la última fase, se utilizaron como herramientas de evaluación un grupo de discusión con los docentes y otra prueba de comprensión con los estudiantes, a fin de conocer los avances y dificultades en la aplicación de la propuesta.

Como técnica de análisis de los datos obtenidos durante la investigación se utilizaron dos matrices de categorización. La primera de ellas con el fin de establecer las relaciones existentes entre algunas variables tales como comprensión de lectura, factores intrínsecos a los estudiantes que dificultan la comprensión, factores extrínsecos a los estudiantes que dificultan la comprensión, factores pedagógicos y uso de herramientas didácticas. La segunda matriz hace referencia a la observación en aula y la comparación de los hallazgos durante la fase de diagnóstico y la de la evaluación, de manera que sea posible determinar la presencia de dificultades específicas antes y después de la intervención pedagógica.

La planeación de las estrategias de intervención se organizó en cuatro unidades didácticas, cada unidad alrededor de un tipo de texto (narrativo, informativo, explicativo y argumentativo). Se consideró el proceso que implica la prelectura, lectura y poslectura, al promover estrategias antes, durante y después de leer en cada unidad, con el fin de que tanto maestros como estudiantes reconozcan acciones de tipo general que son válidas para el trabajo de la comprensión lectora, no solo en el nivel de primaria, sino en los diferentes momentos que se quieran trabajar.

\section{Resultados}

Los hallazgos obtenidos a través de cada una de las herramientas de recolección de datos utilizada permitieron conocer cómo los mismos niños son conscientes de que existen factores internos y externos a ellos que imposibilitan una adecuada comprensión 
de los textos que se manejan dentro y fuera de las aulas. Ejemplo de lo anterior es que un alto porcentaje de estudiantes realiza lecturas porque las considera necesarias, pero no encuentra una motivación personal para llevar a cabo esta actividad. Además, se relaciona la lectura solo con la clase de español, en la que al parecer se emplean textos predominantemente narrativos, lo cual conduce a la carencia de estrategias de lectura eficientes en otras tipologías textuales. Asimismo, los estudiantes reconocen que existen dificultades de diferente tipo cuando tratan de comprender un texto, y en su mayoría las relacionan principalmente con aspectos de una decodificación deficiente. Esto tal vez porque consideran que aún no poseen un adecuado desarrollo de esta habilidad, así se encuentren en los grados finales del nivel de primaria. Solo un porcentaje mínimo puede señalar dificultades externas a ellos, relacionadas con el interés que generan las acciones pedagógicas de los docentes, lo cual se explica porque las estrategias de evaluación de lectura han girado en torno a lo que demuestra haber aprendido el niño, y no frente a la efectividad de la acción pedagógica del maestro.

Por otra parte, la percepción del docente acerca de las dificultades de comprensión se relaciona con dos aspectos fundamentales. El primero es la carencia de habilidades e interés por parte de los estudiantes; el segundo, la falta de apoyo por parte de la familia, ya que no se manifiestan factores que se relacionen con la metodología o el contexto escolar de los niños.

Frente a los aspectos metodológicos propios de cada maestro, estos se relacionan con hechos externos tales como el material que se posee o el hecho de trabajar en un aula multigrado, pues se considera que las acciones pedagógicas que hasta el momento se han implementado apuntan a la promoción de la comprensión. No obstante, sí se reconoce que hay otras posibilidades que podrían ser efectivas para superar las dificultades que se presentan, y se señala en este sentido la importancia de una planeación que incluya variadas estrategias en las que se trabaje desde todas las áreas o asignaturas que maneja el estudiante.

En cuanto al tipo de texto utilizado, el maestro identifica que los textos narrativos son aquellos con los que menos dificultad se presenta, pero no se demuestra que se manejen otros tipos de texto. Incluso, no se emplean términos para definir una tipología textual, ni se tienen en cuenta a la hora de definir las estrategias que es necesario fortalecer; razón por la cual el uso de varios tipos de textos es un aspecto fundamental a tener en cuenta en la elaboración de la propuesta pedagógica.

En los niveles de comprensión textual, se encontró una variación positiva en el texto inferencial y el intertextual. En la prueba de diagnóstico, el nivel literal presentó el mejor desempeño, lo cual generó confianza en los maestros, considerando que este aspecto no era una dificultad marcada. Por esta razón, durante la aplicación se descuidó el trabajo en este nivel. En cuanto al nivel crítico, se pudo observar que los maestros quisieron trabajarlo de la mejor forma, pero aún no se tienen claras las preguntas y acciones que motiven un pensamiento crítico.

Asimismo, en la creación de unidades didácticas en trabajo colaborativo con los maestros se buscó utilizar una tipología textual variada, la cual llamara la atención de los estudiantes y a su vez permitiera desarrollar procesos de pensamiento que beneficien su comprensión lectora. Los tipos de texto que se emplearon son los propuestos por los "Lineamientos Curriculares". Dado que dicha clasificación se realizó con una intención pedagógica, no se hizo uso del texto argumentativo en la prueba de comprensión, ya que su objetivo principal es una toma de posición, razón por la cual se trabajó en el desarrollo de las unidades didácticas.

Desde la etapa de diagnóstico, los docentes y estudiantes manifestaron que los textos narrativos son aquellos que comprenden mejor, de manera que durante la intervención pedagógica se introdujeron otros tipos de narraciones, tales como la historieta y el guion. Esto fortaleció el análisis de secuencias textuales, personajes y estructura narrativa, lo cual se manifiesta en el índice de mejoramiento identificado. Algo similar ocurre con el texto informativo, más cercano a las aulas y al contexto escolar. En el texto explicativo no se muestran avances, pues en las acciones pedagógicas de la propuesta se hace uso de textos tales como la receta y las reglas de juego, las cuales resultaron de difícil comprensión para los niños debido a su poco uso en el contexto académico.

Como resultado principal del proceso de investigación se estructuró un documento que sintetiza aspectos teóricos y prácticos del diseño de la propuesta pedagógica, a fin de fortalecer la comprensión textual de los grados $4 .^{\circ}$ y $5 .^{\circ}$. Se trata de una guía para el docente de básica primaria, la cual pretende mostrar algunas posibilidades de trabajo en el aula a partir de la información recolectada y el valioso aporte de los docentes participantes. La guía del docente se divide en cuatro capítulos que son fruto 
de la fundamentación teórica y metodológica de esta investigación. En ella se presentan estrategias generales de comprensión y las unidades didácticas que se trabajaron alrededor de los textos de tipo narrativo, explicativo, informativo y argumentativo. Estas unidades fueron aplicadas por los docentes y se fortalecieron a partir de los resultados obtenidos, evidenciados a través de la prueba de comprensión, de evaluación, el grupo de discusión y la observación en el aula por parte de las investigadoras.

\section{Discusión}

El proceso investigativo que se abordó en el estudio "El mundo de la lectura: estrategias para la comprensión”, se desarrolló a través de una serie de fases de investigación. La primera fase, destinada al diagnóstico del nivel de comprensión de lectura, se llevó a cabo con un grupo de estudiantes de los grados cuarto y quinto, de la zona rural y urbana del municipio de Machetá, en Cundinamarca, Colombia. Es importante resaltar la evaluación de tipo de diagnóstico como primera etapa del proceso, ya que en muchos de los casos esta actividad no se lleva a cabo de manera formal en el aula, sino que se identifican las dificultades mediante observación, lo cual no permite identificar específicamente las tareas de comprensión en las que se debe trabajar.

Gracias a la información recolectada durante el diagnóstico, se identificaron dificultades puntuales que presentaron la mayor parte de las estudiantes, relacionadas principalmente con el reconocimiento de la intención comunicativa, comparación de diferentes tipos de textos, proposición de hipótesis predictivas, descripción de personajes, acciones y espacios de los textos literarios, identificación de estructuras textuales e inferencia de información no explícita en el texto. Las dificultades en comprensión textual se encuentran con frecuencia en poblaciones que tienen poca accesibilidad a recursos de información actualizados, y en las cuales la tarea docente se ve obstaculizada por factores internos y externos a su actividad. Tal como se mostró en el desarrollo de la investigación, es común que los docentes encuentren que a sus estudiantes se les dificulta la comprensión de los textos debido a aspectos tales como indisciplina, dificultades en atención y falta de recursos didácticos, todo lo cual requiere de acciones por parte del docente a fin de minimizarlos.

Sin embargo, en este punto se debe reconocer que existen algunas actitudes fuera de la pedagogía del docente que obstaculizan la enseñanza-aprendizaje. Dichas actitudes se relacionan con la desmotivación por parte del docente, exigencias de la institución, planes de estudio extensos y la falta de una planeación transversal que integre las áreas académicas.

Estas razones permitieron que durante la investigación se realizara la planeación y puesta en marcha de una propuesta pedagógica, organizada mediante cuatro unidades didácticas a partir de las fallas encontradas, y en la cual el docente asumió la responsabilidad de autoevaluarse, de manera constructiva y productiva, con el propósito de mejorar las habilidades comprensivas de sus estudiantes. De esta forma se vincula de forma colaborativa con el investigador en la construcción de posibles soluciones, prácticas y didácticas que se presentaron a los estudiantes.

Un aspecto valioso de la intervención fue la vinculación de las diferentes áreas de conocimiento, pues los mismos docentes reconocen la transversalidad de la lectura, confirmando lo expuesto por Solé: "La enseñanza de la lectura no es cuestión de un curso o un profesor, sino que es una cuestión de escuela, de proyecto curricular y de todas las materias en las que interviene" (14).

Esta intervención permitió - si no en su totalidad- contribuir en un alto porcentaje al mejoramiento de la comprensión de lectura, basada en la puesta en marcha de actividades previamente planeadas y ensambladas en unidades didácticas, las cuales elaboraron maestros e investigadores. De la misma forma, se confirma que el uso de variedad de textos en el aula genera expectativa en los estudiantes, ya que permite abarcar una multitud de temas de interés a través de textos informativos, explicativos y argumentativos, de tal forma que se supere el uso exclusivo de textos narrativos en las acciones de comprensión textual que, generalmente, se promueven en clase.

Aun cuando se evidenciaron cambios positivos en algunas prácticas de los maestros - reflejadas en los resultados de los estudiantes-, es necesario anotar que las verdaderas transformaciones se darán a través de un largo proceso, en el cual el docente es el protagonista principal. Esto en la medida en que se continúe el trabajo de formulación y aplicación de estrategias efectivas para la comprensión textual. De acuerdo con Furman, citado por Duque, los verdaderos cambios en las prácticas de aula requieren de cuatro condiciones:

1. Que el docente se convenza de que debe cambiar dado que sus prácticas no son efectivas (prueba de ineficacia). 
2. Que el docente experimente otras formas de enseñar y aprender (prueba de existencia).

3. Que el docente se dé cuenta de que lo puede hacer (prueba de capacidad).

4. Que el tiempo requerido para desarrollar y consolidar nuevas prácticas es de al menos entre dos a tres años (sostenibilidad). (4)

Es así como en el desarrollo de la propuesta pedagógica se han dado las tres primeras condiciones, al mostrarle al docente la necesidad de algunos cambios y posibles formas de hacerlo. De esta manera queda bajo responsabilidad del maestro y de la institución la sostenibilidad de las estrategias.

El éxito en la eficacia de la intervención pedagógica estuvo determinado en gran medida por la apropiación por parte de los estudiantes de las estrategias que se trabajaron, con miras a ser aplicadas en los diferentes momentos en que se realice la lectura. De acuerdo con Trabasso y Bouchard, citados por Gutiérrez-Braojos y Salmerón:

Específicamente en tareas de comprensión de texto, las estrategias de aprendizaje se interpretan como una toma de decisiones sobre la selección y uso de procedimientos de aprendizaje que facilitan una lectura activa, intencional, autorregulada y competente en función de la meta y las características del material textual. (185)

Esto llama la atención sobre la continuidad de los procesos de intervención pedagógica, hasta llegar a que sea el estudiante quien emplee por su propia iniciativa una y otra estrategia para garantizar la comprensión de un texto.

En la implementación de la propuesta se hizo énfasis en las estrategias de tipo general antes, durante y después de la lectura. Sin embargo, hay que aclarar que la gama de estrategias es amplia y su aplicación depende de la experticia de cada docente. Según Gutiérrez-Braojos y Salmerón, es posible reconocer estrategias cognitivas, metacognitivas, motivacionales-afectivas y contextuales, siempre y cuando el lector las utilice de forma intencional y con una meta de comprensión definida, lo cual no se logra en un periodo de tiempo corto, sino con la continuidad y constancia de los procesos de apoyo por parte de los docentes. Esto en razón a que su compromiso determina en gran medida el resultado de las acciones pedagógicas que desarrollan.
A través de la investigación se pudo observar que en aquellas sedes que contaban con maestros que desarrollaron las unidades didácticas en los tiempos y de acuerdo con la planeación prevista, los resultados fueron positivos en relación con el mejoramiento $\mathrm{y}$ avance en la comprensión lectora de los estudiantes. En contraste, se presentaron resultados bajos con estudiantes que no completaron el desarrollo de las unidades debido a que algunos docentes no tuvieron en cuenta la planeación. En consecuencia, cuando el estudiante está bajo la tutoría de docentes que asumen su rol de manera responsable y propositiva, sus desempeños son mejores.

\section{Conclusiones}

Las dificultades alrededor del proceso de comprensión de lectura de los estudiantes están vinculadas a factores extrínsecos, tales como la metodología de sus maestros o la situación socioambiental y familiar. De igual forma, a factores intrínsecos tales como motivación, procesos psicológicos básicos y cognitivos, frente a las cuales los maestros deben aplicar estrategias grupales ya que las características del contexto no permiten un trabajo de carácter específico. Esto se debe a que cada una de las sedes rurales es multigrado o unitaria, y la sede urbana cuenta con más de 30 estudiantes en cada grupo, lo cual disminuye las posibilidades en la eficacia de los procesos de enseñanza y exige un mayor esfuerzo y compromiso por parte del docente.

Una barrera para que se logren mejores resultados la constituye el que los maestros consideran que sus estudiantes son los responsables de su motivación frente a la lectura y el aprendizaje. Desconocen muchas veces de esta manera las falencias en aspectos metodológicos, lo cual genera un escaso compromiso de los estudiantes en la aplicación de sus conocimientos.

De la misma forma, aunque los estudiantes reconocen la importancia de la lectura para el desarrollo de sus actividades académicas, no la consideran un aprendizaje necesario en su vida cotidiana, ni una actividad que les genere altos niveles de motivación o interés propio. La perciben solo como un requisito para lograr metas académicas, de manera que queda en manos del maestro la labor de relacionar la actividad de la escuela con la realidad del contexto del estudiante, para así garantizar que el aprendizaje no sea de momento. 
Aspectos tales como la didáctica son fundamentales en la creación de estrategias, las cuales les permitan a los estudiantes de los grados $4 .^{\circ}$ y $5 .^{\circ}$ lograr una comprensión de lectura de todos los textos presentados de acuerdo con el área y su nivel de desarrollo. Es por eso que las unidades didácticas permiten alcanzar objetivos específicos frente al proceso lector y de comprensión de los estudiantes, vinculando aspectos relacionados con las etapas de lectura y su sistematización, así como la integración con vivencias personales y sociales desde el ámbito rural y urbano, lo cual logra motivar al estudiante a indagar su propia realidad.

Las estrategias pedagógicas que se incluyen en las unidades didácticas permiten un abordaje seleccionado de cada tipología textual y un abordaje desde el cuestionamiento, la lúdica, la reflexión y la creatividad, al utilizarlas en diferentes momentos de la lectura: antes, durante y después. Inicialmente, como sucedió con el grupo participante en la investigación, pareciera ser una tarea ardua y dispendiosa, pero una vez el docente se compromete con su construcción se da cuenta de la utilidad de las mismas con sus grupos de trabajo.

De acuerdo con los resultados obtenidos, se ve que en los grados cuarto y quinto es necesario fortalecer el uso de textos explicativos, informativos y argumentativos que contengan temáticas que llamen la atención de los niños. Esto con el propósito de trabajar los procesos de pensamiento, empleados no solo para decodificar un texto, sino para generar una verdadera interacción entre el lector y el texto, así como descubrir la información, el contexto y la intención que quiere transmitir el autor.

La construcción cooperativa de recursos didácticos y pedagógicos que incluya la metodología utilizada en cada sesión académica con los estudiantes, permite que el docente adquiera un compromiso y una disposición más activa frente a la consecución de logros dentro y fuera del aula de clase, al realizar un intercambio de experiencias con sus pares, lo cual indudablemente lo lleva a mejorar sus propias prácticas de aula.
Reconocer las dificultades que poseen los estudiantes en el proceso de comprensión de lectura ya sea fisiológico, psicológico o de metodología-, le permite al docente y al padre de familia realizar un procedimiento adecuado acompañado de otros profesionales. Aunque hay que partir de reconocer que los estudiantes, de acuerdo con su desarrollo cognitivo e influenciados por factores del contexto, logran desarrollar habilidades en torno a la comprensión de lectura, es una labor común entre padres, profesores, compañeros de aula y el estudiante mismo la construcción de estrategias que le permitan a este último un mayor avance en el área de comprensión.

\section{Referencias}

Arias, Juan de Dios. Problemas de aprendizaje. Bogotá: Universidad Pedagógica Nacional. Red de cualificación de Educadores en Ejercicio, 2003. Impreso.

Bautista, Nelly. Proceso de la investigación cualitativa: epistemología, metodología y aplicaciones. Bogotá: Manual Moderno, 2001. Impreso.

Duque, Mauricio. Pilares para un programa de desarrollo profesional situado: resumen de hallazgos sobre la formación continua y las transformaciones de prácticas. Bogotá: Universidad de los Andes, 2015. Impreso.

Gutiérrez-Braojos, Calixto y Salmerón, Honorio. "Estrategias de comprensión lectora: enseñanza y evaluación en educación primaria". Revista de Currículum y Formación de Profesorado 16.1 (2012): 183-202. Impreso.

Ministerio de Educación Nacional. Lineamientos curriculares de Lengua Castellana. Bogotá: Editorial Cooperativa, 1998. Impreso.

Mendoza, Antonio, et al. Didáctica de la lengua y la literatura. Madrid: Editorial Prentice Hal, 2003. Impreso.

Pérez, Mauricio. Leer y escribir en la escuela: Algunos escenarios pedagógicos y didácticos para la reflexión. Bogotá: Instituto Colombiano para el Fomento de la educación Superior (ICFES), 2003. Impreso.

Solé, Isabel. Estrategias de lectura. Barcelona: Editorial Graó, 1992. Impreso. 